\title{
Photoemission spectra of massless Dirac fermions on the verge of exciton condensation
}

\author{
Stefan Rist, ${ }^{1}$ A.A. Varlamov, ${ }^{2}$ A.H. MacDonald, ${ }^{3}$ Rosario Fazio, ${ }^{4}$ and Marco Polini ${ }^{5}$ \\ ${ }^{1}$ CNR-SPIN, Corso Perrone 24, I-16152 Genova and NEST, Scuola Normale Superiore, I-56126 Pisa, Italy \\ ${ }^{2}$ CNR-SPIN, Tor Vergata, Viale del Politecnico 1, I-00133 Rome, Italy \\ ${ }^{3}$ Department of Physics, University of Texas at Austin, Austin, Texas 78712, USA \\ ${ }^{4}$ NEST, Scuola Normale Superiore and Istituto di Nanoscienze-CNR, I-56126 Pisa, Italy \\ ${ }^{5}$ NEST, Istituto Nanoscienze-CNR and Scuola Normale Superiore, I-56126 Pisa, Italy
}

\begin{abstract}
Angle-resolved photoemission spectroscopy (ARPES) is a powerful probe of electron correlations in two-dimensional layered materials. In this Letter we demonstrate that ARPES can be used to probe the onset of exciton condensation in spatially-separated systems of electrons and holes created by gating techniques in either double-layer graphene or topological-insulator thin films.
\end{abstract}

Introduction-Electron-hole pairs can undergo BoseEinstein condensation forming a macroscopically coherent neutral fluid, the exciton condensate (EC) [1]. EC states have been predicted theoretically in a variety of different systems. Spatially separated electrons and holes located on two nearby layers (like those that can be created in GaAs/AlGaAs semiconductor double quantum wells) were considered first some time ago [5]. These double-layer exciton condensates (DLECs) possess spontaneous inter-layer coherence and support counter-flow supercurrents. The DLEC has been discussed more recently in the context of quantum Hall bilayers at total filling factor $\nu_{\mathrm{T}}=1$ 6 9 . The first clear experimental evidence for exciton condensation was obtained a decade ago in quantum Hall bilayer DLECs [10, long after the state was predicted.

There is an ongoing effort to find new systems which display exciton condensation. In addition to the fundamental interest in understanding when this type of order occurs in nature, there is a practical interest in finding ECs that occur under less extreme physical conditions, in particular in the absence of strong magnetic fields and, possibly, at non-cryogenic temperatures. Very recently exciton condensation has been predicted to occur in double-layer graphene [11-14] and in threedimensional (3D) gated topological insulators (TIs) [15. In the TI case spontaneous coherence is established between topologically protected single-particle states on top and bottom surfaces. Both double-layer graphene and TI thin-film systems are described at low energies by two massless Dirac fermion (MDF) Hamiltonians which interact. Since MDF systems are gapless and truly twodimensional (2D), the field-driven carrier densities that can be achieved are much larger than in the semiconductor case. Weaker dielectric screening and linearly dispersive conduction and valence bands help to increase both interaction and disorder energy scales. Finally, MDF bands are perfectly particle-hole symmetric, guaranteeing the almost complete nesting between conduction- and valence-band Fermi surfaces, which favors the coherent state.

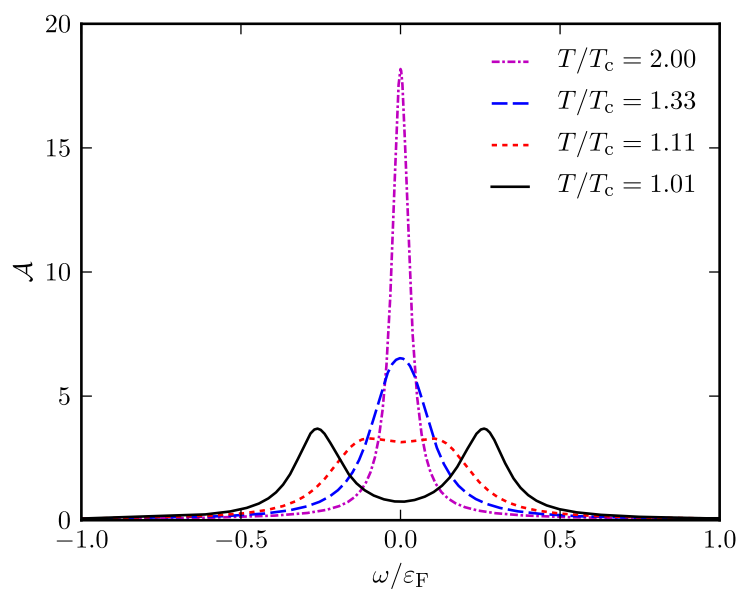

FIG. 1: (Color online) The spectral function of a masslessDirac-fermion double-layer system on the verge of exciton condensation. The spectral function $\mathcal{A}(\boldsymbol{k}, \omega)$ (in units of $1 / \varepsilon_{\mathrm{F}}$, where $\varepsilon_{\mathrm{F}}$ is the Fermi energy) evaluated at $|\boldsymbol{k}|=k_{\mathrm{F}}$ is plotted as a function of $\omega / \varepsilon_{\mathrm{F}}$ for different values of $T_{\mathrm{c}} / T$. The single peak at the Fermi energy in the high-temperature limit splits into two peaks as $T \rightarrow T_{\mathrm{c}}$ from above. All curves have been evaluated by setting $T=0.1 T_{\mathrm{F}}$.

Graphene double-layer systems can be realized by separating two graphene layers by a dielectric [16] (such as $\mathrm{Al}_{2} \mathrm{O}_{3}$ ) or by a few layers of a one-atom-thick insulator such as h-BN [17-20. In both cases inter-layer hybridization is negligible and the nearby graphene layers are, from the point of view of single-particle physics, isolated. Topologically-protected massless Dirac 2D electron systems (MD2DESs) appear automatically [21] at the top and bottom surfaces of a 3D TI thin film. 3D TIs in a slab geometry offer two surface states that can be far enough apart to make single-electron tunneling negligible, but close enough for Coulomb interactions between surfaces to be important.

We report on an analysis undertaken in support of the experimental quest for ECs with MDF character. Because of the potential value of probes which can detect incipient order while still above the critical temperature 
$T_{\mathrm{c}}$, we examine the influence of pairing fluctuations on the ARPES spectra that are commonly used to probe two-dimensional systems like graphene. Fluctuation corrections to thermodynamic and transport observables have previously been extensively studied in superconductors [22]. General considerations based on the uncertainty principle allow one to conclude that, like Cooper pairs in superconductors, excitons have a finite lifetime above $T_{\mathrm{c}}$ which is inversely proportional to the distance from the critical temperature. Exciton fluctuations will therefore influence the behavior of the double layer providing distinct signatures in the vicinity of the EC instability. Mink et al. 23, for example, have investigated the role of fluctuations in a Coulomb-drag transport setup, demonstrating that the drag resistivity grows logarithmically upon lowering the temperature towards $T_{\mathrm{c}}$.

Experimental evidence for ECs in double-layer systems has so far been largely based on transport measurements. In the case of quantum Hall systems the transport phenomenology [8 10, 24] is spectacular in separately contacted coherent double layers. In this Letter we study the effect of fluctuations on a contactless observable: the one-body spectral function, which can be accessed by ARPES. ARPES, a photon-in electron-out experiment [25], has been established as an extremely important tool in studying the quasiparticle dynamics in graphene [26] and provided the first direct evidence for conducting surface states in 3D TIs 27.

The quantity measured in ARPES is the one-body spectral function $\mathcal{A}(\boldsymbol{k}, \omega)$. A normal Fermi liquid is characterized by a spectral function that, for momenta $|\boldsymbol{k}|$ close to the Fermi wave number $k_{\mathrm{F}}$, has a single Lorentzian peak as a function of frequency with a width that vanishes as $\left(k-k_{\mathrm{F}}\right)^{2}$ [28. Below we demonstrate that the spectral function and its integral over $\boldsymbol{k}$, i.e. the density-of-states (DOS) $\nu(\omega)$, show precursors of the DLEC phase of unhybridized MD2DESs at temperatures larger than the transition temperature. The peak in $\left.\mathcal{A}(\boldsymbol{k}, \omega)\right|_{|\boldsymbol{k}|=k_{\mathrm{F}}}$ broadens as one approaches the transition and splits into two peaks, as can be seen in Fig. 1. At the same time $\nu(\omega)$ develops a strong dip at the Fermi energy in contrast to the featureless behavior displayed by the non-interacting system (Fig. 33).

MDF Hamiltonian and exciton condensation-We consider a double layer hosting MDFs whose top (bottom) layer is p-doped (n-doped). The two layers are coupled only via the Coulomb interaction between the carriers and electron tunneling is completely suppressed. Following Refs. [11-13, we include in our calculations only the conduction band (upper Dirac cone) for the n-doped layer and the valence band (lower Dirac cone) for the pdoped layer. This is justified since the temperatures we consider are sufficiently small and the screening lengths sufficiently large, so that the energetically remote bands can, in first instance, be considered as inert. The band dispersions can be approximated by $\xi_{\mathrm{b}}(\boldsymbol{k})=v|\boldsymbol{k}|-\varepsilon_{\mathrm{F}}$ and

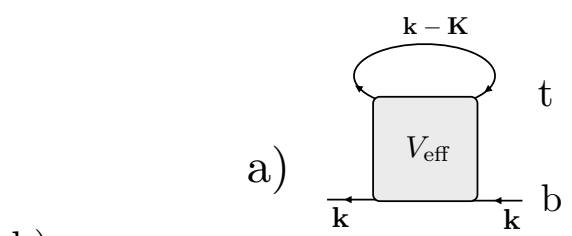

b)

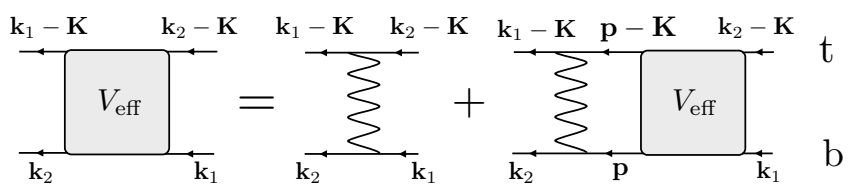

FIG. 2: a) The diagram considered for the self energy: the upper line refers to the top (hole) layer while the lower line to the bottom (electron) layer. b) The ladder approximation for the effective interaction $V_{\text {eff }}-$ Eq. (2). The thin wavy line is the screened interlayer interaction $V_{0}\left(\boldsymbol{k}_{1}, \boldsymbol{k}_{2}, \boldsymbol{K}\right) ; \boldsymbol{K}$ is the center-of-mass momentum and $\boldsymbol{p}$ is an integration variable.

$\xi_{\mathrm{t}}(\boldsymbol{k})=-v|\boldsymbol{k}|+\varepsilon_{\mathrm{F}}=-\xi_{\mathrm{b}}(\boldsymbol{k})$ for the bottom (b) and top (t) layers, respectively. Here $v$ is the Dirac velocity, which is $\approx 10^{6} \mathrm{~m} / \mathrm{s}$ for double-layer graphene and $\approx 5 \times 10^{5} \mathrm{~m} / \mathrm{s}$ for the surface states of typical known TI thin films [29]. We set $\hbar=1$ throughout this work and include only inter-layer interactions, assuming that intra-layer interactions can be taken into account by renormalizing the bare parameters of the two separate-layer Hamiltonians.

We therefore consider the following effective Hamiltonian:

$$
\begin{aligned}
\hat{\mathcal{H}} & =\sum_{\boldsymbol{k}, i=\mathrm{b}, \mathrm{t}} \xi_{i}(\boldsymbol{k}) \hat{c}_{i, \boldsymbol{k}}^{\dagger} \hat{c}_{i, \boldsymbol{k}} \\
& +\sum_{\boldsymbol{k}_{1}, \boldsymbol{k}_{2}, \boldsymbol{K}} V_{0}\left(\boldsymbol{k}_{1}, \boldsymbol{k}_{2}, \boldsymbol{K}\right) \hat{c}_{\mathrm{t}, \boldsymbol{k}_{1}-\boldsymbol{K}}^{\dagger} \hat{c}_{\mathrm{b}, \boldsymbol{k}_{2}}^{\dagger} \hat{c}_{\mathrm{b}, \boldsymbol{k}_{1}} \hat{c}_{\mathrm{t}, \boldsymbol{k}_{2}-\boldsymbol{K}}
\end{aligned}
$$

where $\hat{c}_{i, \boldsymbol{k}}\left(\hat{c}_{i, \boldsymbol{k}}^{\dagger}\right)$ is the annihilation (creation) operator for an electron in the $i$-th layer with momentum $\boldsymbol{k}$. The bare (repulsive) interaction $V_{0}\left(\boldsymbol{k}_{1}, \boldsymbol{k}_{2}, \boldsymbol{K}\right)$ depends on the three wave vectors $\boldsymbol{k}_{1}, \boldsymbol{k}_{2}$, and $\boldsymbol{K}$ (and not only on $\left|\boldsymbol{k}_{1}-\boldsymbol{k}_{2}\right|$, which is the momentum transfer) because of the chirality factors [30, which encode the suppressed backscattering property of MDFs. After a particle-hole transformation $\hat{c}_{\mathrm{t}, \boldsymbol{k}} \rightarrow \hat{h}_{\mathrm{t},-\boldsymbol{k}}^{\dagger}$ in the top layer one immediately sees that the second term in Eq. (1) describes pairing between an electron with momentum $\boldsymbol{k}_{1}$ in the bottom layer and a hole with momentum $-\boldsymbol{k}_{1}+\boldsymbol{K}$ in the top layer, interacting via an attractive potential equal to $-V_{0}\left(\boldsymbol{k}_{1}, \boldsymbol{k}_{2}, \boldsymbol{K}\right)$.

The EC state is signaled by a pole in the finitetemperature many-particle scattering amplitude $V_{\text {eff }}$ which appears at a critical temperature $T=T_{\mathrm{c}}$ when the center-of-mass momentum $\boldsymbol{K}$ of a pair and the "conjugate" energy $\Omega=\xi_{\mathrm{t}}\left(\boldsymbol{K}-\boldsymbol{k}_{1}\right)+\xi_{\mathrm{b}}\left(\boldsymbol{k}_{1}\right)$ vanish. In 
Fig. 2 we show the diagrammatic representation of the electron self energy [panel a)] and the many-particle scattering amplitude in the ladder approximation [panel b)]. The ladder sum in panel b) is analytically intractable for a generic two-body potential $V_{0}\left(\boldsymbol{k}_{1}, \boldsymbol{k}_{2}, \boldsymbol{K}\right)$. To make progress, we approximate $[23] V_{0}\left(\boldsymbol{k}_{1}, \boldsymbol{k}_{2}, \boldsymbol{K}\right)$ by a momentum-independent constant $U$. (As discussed in Ref. [23], close to $T_{\mathrm{c}}$ the chirality factors do not play any qualitatively significant role.) This approximation is reasonable since it relies on screening, which is robust when the electron and hole densities in the two layers are large. Substantial numerical work is needed to transcend this simple approximation and, in any event, a brute-force numerical approach would mask the elementary understanding of the physical effect we want to explain here.

When the bare interaction does not carry momentum labels, the ladder sum in Fig. 2 is a geometric series which can be summed to yield

$$
V_{\mathrm{eff}}\left(\boldsymbol{K}, \Omega_{\nu}\right) \equiv \frac{U}{1-U \Xi\left(\boldsymbol{K}, \Omega_{\nu}\right)} .
$$

Here $\Omega_{\nu}=2 \pi \nu / \beta$ are bosonic Matsubara frequencies with $\beta=\left(k_{\mathrm{B}} T\right)^{-1}$,

$$
\Xi(\boldsymbol{K}, \Omega)=\frac{1}{A} \sum_{\boldsymbol{k}} \frac{n_{\mathrm{F}}\left(\xi_{\mathrm{t}}(\boldsymbol{k}-\boldsymbol{K})\right)-n_{\mathrm{F}}\left(\xi_{\mathrm{b}}(\boldsymbol{k})\right)}{\xi_{\mathrm{b}}(\boldsymbol{k})-\xi_{\mathrm{t}}(\boldsymbol{k}-\boldsymbol{K})-\mathrm{i} \Omega}
$$

is a pairing susceptibility, $A$ is the $2 \mathrm{D}$ electron system area , and $n_{\mathrm{F}}(E)=1 /[1+\exp (\beta E)]$ is the Fermi-Dirac distribution. The pairing susceptibility evaluated at $\boldsymbol{K}=\mathbf{0}$ and $\Omega=0$ diverges logarithmically at zero temperature, and therefore the denominator of Eq. (2) must vanish at some non-zero temperature $T_{\mathrm{c}}$ no matter how small the electron-hole attraction. This is the mean-field pairing transition temperature [31. As temperature decreases towards $T_{\mathrm{c}}$, pairing fluctuations first lead to an enhance- ment of $V_{\text {eff }}$ for small $\boldsymbol{K}$ and $\Omega$ and ultimately at $T=T_{\mathrm{c}}$ to a pole at $\Omega=0$ and $\boldsymbol{K}=\mathbf{0}$.

Exciton fluctuations and ARPES spectra. - The anomalous properties of the effective interaction $V_{\text {eff }}$ on approaching $T_{\mathrm{c}}$ lead to observable consequences [22]. Here we concentrate on the spectral function of the bottom (n-doped) layer $\mathcal{A}_{\mathrm{b}}(\boldsymbol{k}, \omega)=-\pi^{-1} \Im m G_{\mathrm{b}}^{\mathrm{R}}(\boldsymbol{k}, \omega)$, which can be calculated from the retarded self-energy $\Sigma_{\mathrm{b}}^{\mathrm{R}}(\boldsymbol{k}, \omega)$. As is customary [22], we proceed by first evaluating the self-energy on the imaginary-frequency axis and then analytically continue it to real frequency. According to the diagram shown in Fig. 2a), the self-energy is given by

$\Sigma_{\mathrm{b}}\left(\boldsymbol{k}, \omega_{n}\right)=-\frac{1}{\beta A} \sum_{\boldsymbol{K}, \Omega_{\nu}} V_{\mathrm{eff}}\left(\boldsymbol{K}, \Omega_{\nu}\right) G_{\mathrm{t}}^{(0)}\left(\boldsymbol{k}-\boldsymbol{K}, \omega_{n}-\Omega_{\nu}\right)$

where $\omega_{n}=2 \pi(n+1 / 2) / \beta$ are fermionic Matsubara frequencies and $G_{\mathrm{t}}^{(0)}\left(\boldsymbol{k}, \omega_{n}\right)=\left[\mathrm{i} \omega_{n}-\xi_{\mathrm{t}}(\boldsymbol{k})\right]^{-1}$ is the freeelectron Green's function in the top layer. In order to calculate the self-energy from Eq. (4) we approximate $V_{\text {eff }}$ by a form valid [22] at small $\boldsymbol{K}$ and $\Omega$ where fluctuations are strongest:

$$
V_{\mathrm{eff}}^{\mathrm{R}}(\boldsymbol{K}, \Omega) \approx \frac{8 /\left(\pi \beta \nu_{0}\right)}{-\mathrm{i} \Omega+\alpha(T)+8 \xi_{0}^{2} \boldsymbol{K}^{2} /(\pi \beta)},
$$

where $\alpha(T)=8 \log \left(T / T_{\mathrm{c}}\right) /(\pi \beta)$ measures the distance from the critical point, $\xi_{0}=\left[7 \zeta(3) v^{2} \beta^{2}\right] /\left(32 \pi^{2}\right)$ is the coherence length, and $\nu_{0}=\varepsilon_{\mathrm{F}} /\left(2 \pi v^{2}\right)$ is the single-particle DOS at the MDF Fermi energy. Using Eq. (5) for the retarded fluctuation propagator we can then perform the sum over the Matsubara frequencies in Eq. (4). After analytic continuation we obtain the following expression for the imaginary part of the retarded self energy:

$$
\Im m \Sigma_{\mathrm{b}}^{\mathrm{R}}(\boldsymbol{k}, \omega)=\frac{4}{\pi \beta \nu_{0}} \int \frac{d^{2} \boldsymbol{q}}{(2 \pi)^{2}} \frac{\omega+\xi_{\mathrm{b}}(\boldsymbol{q})}{\left(\omega+\xi_{\mathrm{b}}(\boldsymbol{q})\right)^{2}+\gamma(\boldsymbol{k}-\boldsymbol{q})^{2}}\left[\tanh \left(\frac{\beta \xi_{\mathrm{b}}(\boldsymbol{q})}{2}\right)-\operatorname{coth}\left(\frac{\beta\left(\omega+\xi_{\mathrm{b}}(\boldsymbol{q})\right)}{2}\right)\right],
$$

where $\gamma(\boldsymbol{q})=\alpha(T)+8 \xi_{0}^{2} \boldsymbol{q}^{2} /(\pi \beta)$.

Once the imaginary part of the self-energy is known, the real part can be calculated via the Kramers-Kronig relation [28, 35]

$$
\Re e \Sigma_{\mathrm{b}}^{\mathrm{R}}(\boldsymbol{k}, \omega)=\Re e \Sigma_{\mathrm{b}}^{\mathrm{R}}(\boldsymbol{k}, \infty)+\mathcal{P} \int \frac{d \nu}{\pi} \frac{\Im m \Sigma_{\mathrm{b}}^{\mathrm{R}}(\boldsymbol{k}, \nu)}{\nu-\omega} .
$$

Since $\Re e \Sigma_{\mathrm{b}}^{\mathrm{R}}(\boldsymbol{k}, \infty)$ depends weakly on momentum $\boldsymbol{k}$ near $k_{\mathrm{F}}$ and on temperature it can be absorbed by measuring energies relative to the Fermi energy. In practice we compute the self energy by numerical integration of
Eq. (6) and Eq. (7), neglecting $\Re e \Sigma_{\mathrm{b}}^{\mathrm{R}}(\boldsymbol{k}, \infty)$ in the latter. In the weak-coupling limit we find $\mathcal{A}_{\mathrm{b}}(\boldsymbol{k}, \omega)$ to be a delta function peaked at some frequency $\Delta \omega$. Finally, we calculate $\mathcal{A}_{\mathrm{b}}(\boldsymbol{k}, \omega)$ for various values of $T / T_{\mathrm{c}}$ by using $\Re e \Sigma_{\mathrm{b}}^{\mathrm{R}}(\boldsymbol{k}, \infty)=-\Delta \omega$. The parameter we change in order to approach the phase transition is thus the interaction strength $U$ or, equivalenty, $T_{\mathrm{c}}$.

Numerical results - In Fig. 1 we plot the sum of the spectral functions of top and bottom layers, $\mathcal{A}(\boldsymbol{k}, \omega)=$ $\mathcal{A}_{\mathrm{b}}(\boldsymbol{k}, \omega)+\mathcal{A}_{\mathrm{t}}(\boldsymbol{k}, \omega)$. Due to the perfect particle-hole symmetry of our model, the spectral function of the top (hole- 


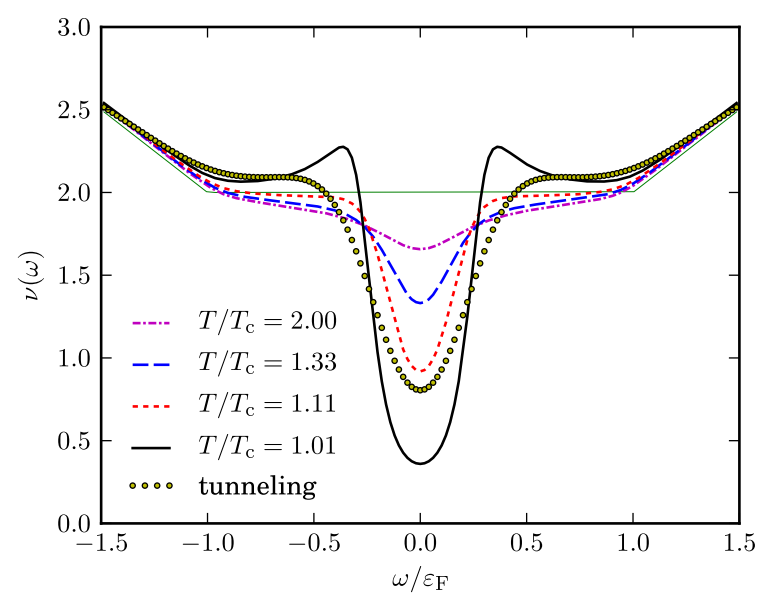

FIG. 3: (color online) The density-of-states $\nu(\omega)$ (in units of the non-interacting value $\nu_{0}$ ) as a function of $\omega$ (in units of and measured from the Fermi energy $\varepsilon_{\mathrm{F}}$ ). The color coding and labeling is identical to Fig. 11 The filled (yellow) circles denote the convolution on the r.h.s. of Eq. (9) evaluated at $T / T_{\mathrm{c}}=1.01$.

doped) layer $\mathcal{A}_{\mathrm{t}}(\boldsymbol{k}, \omega)$ is given by $\mathcal{A}_{\mathrm{t}}(\boldsymbol{k}, \omega)=\mathcal{A}_{\mathrm{b}}(\boldsymbol{k},-\omega)$. In Fig. 1 we illustrate the dependence of $\mathcal{A}(\boldsymbol{k}, \omega)$ on frequency $\omega$ for $|\boldsymbol{k}|=k_{\mathrm{F}}$. This plot refers to a fixed temperature, $T=0.1 T_{\mathrm{F}}$, and to different values of $T / T_{\mathrm{c}}$. We note that the single Lorenzian peak of the spectral function becomes broader and broader as one approaches the phase transition and eventually splits into two separate peaks very close to the phase transition. Note that the splitting between the two peaks is very large, $\sim 5 k_{\mathrm{B}} T_{\mathrm{c}}$ at $T / T_{\mathrm{c}}=1.01$. The two-peak structure resembles the behavior of the spectral function expected below the transition temperature. From Bogoliubov theory one indeed expects two delta-function peaks at frequencies $\Omega_{ \pm}= \pm|\Delta|$ where $\Delta$ is the excitation spectrum gap.

From the spectral function one can also calculate the $\operatorname{DOS} \nu(\omega)$ :

$$
\nu(\omega)=\int \frac{d^{2} \boldsymbol{k}}{(2 \pi)^{2}} \mathcal{A}(\boldsymbol{k}, \omega) .
$$

We performed the integral numerically after evaluating the integrand for momenta up to $|\boldsymbol{k}|=3 k_{\mathrm{F}}$. Our results are shown in Fig. 3. where, for the sake of comparison, we have also plotted the non-interacting result. We note that as one approaches the phase transition more and more spectral weight is shifted from the Fermi energy $(\omega=0)$ to higher energies. The resulting dip in the DOS around $\omega=0$ can be seen as a precursor of the gap in the excitation spectrum that opens up only in the broken-symmetry phase.
In recent decades the DOS of electronic systems has been measured by using scanning tunneling microscopy (STM), which probes locally to mitigate the influence of inhomogeneities and some types of disorder 36 . For example the zero bias anomaly of disordered metals [37] has been successfully explored in this way. The differential tunneling conductivity is normally well approximated by

$$
\sigma_{\text {tun }}(V)=\frac{d I}{d V} \propto \int_{-\infty}^{\infty}\left[-\frac{\partial n_{\mathrm{F}}(E)}{\partial E}\right] \nu(E+e V) d E
$$

In Eq. 9 $I$ and $V$ are respectively the tunneling current and the bias voltage between the STM tip and the sample. When $\nu(E)$ is smooth on the scale of $k_{\mathrm{B}} T$ the distribution function on the r.h.s. of Eq. (9) acts like a delta function so that $\sigma_{\text {tun }}(V) \propto \nu(V)$ 37]. The situation is very different in the case of STM studies of fluctuating superconductors above $T_{\mathrm{c}}$. In this case the DOS can have structure that is sharp on the scale of the temperature. The tunneling conductivity $\sigma_{\text {tun }}(V)$ then has a broad pseudogap structure (with a maximum at $\left.e V_{\max }=\pi k_{\mathrm{B}} T_{\mathrm{c}}\right)$, resembling the BCS gap below $T_{\mathrm{c}} 38$. This occurs in spite of the predicted [39, 40] sharp singularity in the fluctuation contribution to the DOS close to $T_{\mathrm{c}}$. The tunneling conductivity is much smoother as a function of temperature or voltage $\left(\sigma_{\text {tun }}(0) / \sigma_{\mathrm{N}} \propto\right.$ $\ln \left[T_{\mathrm{c}} /\left(T-T_{\mathrm{c}}\right)\right]$ where $\sigma_{\mathrm{N}}$ is normal conductivity of the tunnel junction) than DOS is as a function of energy. This distinction ultimately reflects the property that the energetically averaged DOS is not altered by order [41. Similar considerations apply to the EC fluctuation contribution to the tunneling conductivity as illustrated in Fig. 3 (circles) where we have plotted the r.h.s. of Eq. (9) at $T / T_{\mathrm{c}}=1.01$.

In summary, we have demonstrated that ARPES and STM can be used to probe the onset of exciton condensation in spatially-separated systems of electrons and holes created by gating techniques in double-layer graphene and topological-insulator thin films. The advantage of these experimental probes is that one does not need to realize independent contacts to two closely-spaced Dirac layers. These probes can be particularly useful in the context of topological-insulator thin films where separate contacting is precluded by hybridization between top and bottom at the sample edges.

Acknowledgments - Work in Pisa was supported by MIUR through the programs "FIRB IDEAS" - Project ESQUI (Grant No. RBID08B3FM) and "FIRB - Futuro in Ricerca 2010" - Project PLASMOGRAPH (Grant No. RBFR10M5BT), and by the EU Grants NANO-CTM, IP-SOLID, STREP-QNEMS, and STREP-GEOMDISS. AAV was supported by the FP7-IRSES program, Grant No. 236947 "SIMTECH". AHM was supported by SWAN, Welch Foundation grant TBF1473, and by DOE Division of Materials Sciences and Engineering grant DEFG03-02ER45958. 
[1] J.M. Blatt, K.W. Böer, and W. Brandt, Phys. Rev. 126, 1691 (1962).

[2] L.V. Keldysh and A.N. Kozlov, Sov. Phys. JETP 27, 521 (1968).

[3] N.B. Brandt and S.M. Chudinov, JLTP 8, 339 (1972).

[4] A.A. Abrikosov, JLTP 10, 3 (1973).

[5] Y.E. Lozovik and V.I. Yudson, JETP Lett. 22, 274 (1975).

[6] H.A. Fertig, Phys. Rev. B 40, 1087 (1989).

[7] X.-G. Wen and A. Zee, Phys. Rev. Lett. 69, 1811 (1992).

[8] J.P. Eisenstein and A.H. MacDonald, Nature 432, 691 (2004).

[9] J.-J. Su and A.H. MacDonald, Nature Phys. 4, 799 (2008).

[10] I.B. Spielman, et al. , Phys. Rev. Lett. 84, 5808 (2000); Phys. Rev. Lett. 87, 036803 (2001).

[11] H. Min et al., Phys. Rev. B 78, 121401(R) (2008).

[12] C.-H. Zhang and Y.N. Joglekar, Phys. Rev. B 77, 233405 (2008).

[13] Y.E. Lozovik and A.A. Sokolik, JETP Lett. 87, 55 (2008).

[14] M.P. Mink et al., Phys. Rev. B 84, 155409 (2011).

[15] B. Seradjeh, J.E. Moore, and M. Franz, Phys. Rev. Lett. 103, 066402 (2009); see also Z. Wang et al., New J. Phys. 14, 063010 (2012).

[16] S. Kim et al., Phys. Rev. B 83, 161401(R) (2011); S. Kim and E. Tutuc, Solid State Commun. 152, 1283 (2012).

[17] C.R. Dean et al., Nature Nanotech. 5, 722 (2010).

[18] L.A. Ponomarenko et al., Nature Phys. 7, 958 (2011).

[19] L. Britnell et al., Science 335, 947 (2012); L. Britnell et al., Nano Lett. 12, 1707 (2012).

[20] R.V. Gorbachev et al., arXiv:1206.6626.

[21] M.Z. Hasan and C.L. Kane, Rev. Mod. Phys. 82, 3045 (2010); X.-L. Qi and S.-C. Zhang, ibid. 83, 1057 (2011).

[22] A. Larkin and A. Varlamov, Theory of fluctuations in superconductors, (Clarendon Press, Oxford, 2004)

[23] M.P. Mink et al., Phys. Rev. Lett. 108, 186402 (2012); for earlier theoretical works on Coulomb drag in electronic systems in the exciton-condensate phase or approaching an exciton-condensate instability see G. Vignale and A.H. MacDonald, Phys. Rev. Lett. 76, 2786 (1996) and BenYu-Kuang Hu, Phys. Rev. Lett. 85, 820 (2000), respectively. For experimental anomalies of the Coulomb drag transresistivity of electron-hole semiconductor bilayers (at zero magnetic field) see A.F. Croxall et al., Phys. Rev. Lett. 101, 246801 (2008) and J.A. Seamons et al., Phys. Rev. Lett. 102, 026804 (2009).

[24] D. Nandi et al., Nature 488, 481 (2012).

[25] A. Damascelli, Z. Hussain, and Z.-X. Shen, Rev. Mod. Phys. 75, 473 (2003).

[26] See, for example, E. Rollings et al., J. Phys. Chem. Solids 67, 2172 (2006); A. Bostwick et al., Nature Phys. 3, 36 (2007); A. Bostwick et al., Solid State Commun. 143, 63 (2007); S.Y. Zhou et al., Nature Mater. 6, 770 (2007);
A. Bostwick et al., Science 328, 999 (2010); D.A. Siegel et al., Proc. Natl. Acad. Sci. (USA) 108, 11365 (2011); A. L. Walter et al. Phys. Rev. B 84, 085410 (2011) and references therein.

[27] D. Hsieh et al., Nature 452, 970 (2008).

[28] G.F. Giuliani and G. Vignale, Quantum Theory of the Electron Liquid (Cambridge University Press 2005)

[29] See e.g. Y. Xia et al., Nature Phys. 5, 398 (2009).

[30] A.H. Castro Neto et al., Rev. Mod. Phys. 81, 109 (2009).

[31] Since we deal with 2D superfluids, the phase transition under consideration is ultimately of Kosterlitz-Thouless (KT) character. Our theory is most sound when the KT transition temperature and $T_{\mathrm{c}}$ are similar, i.e. when the zero-temperature superfluid stiffness is much larger than $T_{\mathrm{c}}$. In massless-Dirac DLEC this condition is satisfied [1] when $T_{\mathrm{c}}$ is very small compared to the Fermi energy. For large layer separations the effective inter-layer interaction strength $U$ which appears in our theory is expected to be strongly reduced by screening and $T_{\mathrm{c}}$ consequently safely smaller than the Fermi temperature 32 34. At small layer separations, on the other hand, it has been suggested [33, 34] that $U$ strengthens as excitonic fluctuations become established and screening is weakened.

[32] M.Y. Kharitonov and K.B. Efetov, Phys. Rev. B 78, 241401 (2008).

[33] I. Sodemann, D.A. Pesin, and A.H. MacDonald, Phys. Rev. B 85, 195136 (2012).

[34] Y.E. Lozovik, S.L. Ogarkov, and A.A. Sokolik, Phys. Rev. B 86, 045429 (2012).

[35] In Eq. (6) we integrate over momentum by using values of $|\boldsymbol{q}|$ up to $q=10 k_{\mathrm{F}}$. Performing the Hilbert transform in Eq. (7) numerically is quite tricky and deserves special care. In the present case we achieve convergence by integrating over the variable $\nu$ in the range $-2 \times 10^{4} \leq \nu \leq 2 \times 10^{4}$

[36] J. Tersoff and D.R. Hamann, Phys. Rev. B 31, 805 (1985); C.J. Chen, Introduction to Scanning Tunneling Microscopy (Oxford University Press, New York, 2008). For recent reviews on STM applied to graphene sheets see e.g. M. Morgenstern, Phys. Status Solidi (b) 248, 2423 (2011) and E.Y. Andrei, G. Li, and X. Du, Rep. Prog. Phys. 75, 056501 (2012). A number of STM studies of TIs have also appeared in the recent literature: see, for example, P. Roushan et al., Nature 460, 1106 (2009); T. Zhang et al., Phys. Rev. Lett. 103, 266803 (2009); Z. Alpichshev et al., Phys. Rev. Lett. 104, 016401 (2010); Y. Okada et al., ibid. 106, 206805 (2011); H. Beidenkopf et al., Nature Phys. 7, 939 (2011) and references therein.

[37] B.L. Altshuler and A.G. Aronov, Solid State Commun. 30, 115 (1979).

[38] B. Sacépé et al., Nature Commun. 1, 140 (2010).

[39] E. Abrahams, M. Redi, and J.W.F. Woo, Phys. Rev. B 1, 208 (1970).

[40] C. DiCastro et al., Phys. Rev. B 42, 10211 (1990).

[41] A.A. Varlamov and V.V. Dorin, Sov. Phys. JETP 57, 1089 (1983). 